\title{
Modelling hyperfine interactions for nuclear g-factor measurements
}

\author{
B. P. McCormick ${ }^{1, *}$, A. E. Stuchbery ${ }^{1, * *}$, A. Goasduff ${ }^{2}$, A. Kusoglu ${ }^{2,3}$, and G. Georgiev ${ }^{1,2}$ \\ ${ }^{1}$ Department of Nuclear Physics, Research School of Physics, The Australian National University, ACT 2601, Australia \\ ${ }^{2}$ CSNSM, CNRS/IN2P3, Université Paris-Sud, UMR8609, F-91405 ORSAY-Campus, France \\ ${ }^{3}$ Department of Physics, Faculty of Science, Istanbul University, Vezneciler/Fatih, 34134, Istanbul, Turkey
}

\begin{abstract}
A promising technique for $g$-factor measurements on short-lived nuclear states utilises the hyperfine fields of free ions in vacuum. To fully utilise this technique the hyperfine interaction must be modelled based on atomic structure calculations. Atomic structure calculations were performed using the most recent release of the General Relativistic Atomic Structure Package, and Monte-Carlo simulations of atomic-decay cascades in highly charged ions were developed. The simulations were used to fit experimental data on excited ${ }^{56} \mathrm{Fe}$ ions recoiling in vacuum with a view to determining the first-excited state $g$ factor, $g\left(2_{1}^{+}\right)$, of ${ }^{56} \mathrm{Fe}$.
\end{abstract}

\section{Introduction}

A powerful probe for nuclear structure study is the magnetic dipole moment, $\mu$. Usually, for excited states, the $g$ factor is the quantity measured, where $\mathrm{g}=\mu / I, I$ being the spin of the state. The $g$ factor provides a way to probe the wavefunction of a single state. It is sensitive to the composition of broken proton vs neutron pairs, and the angular momentum they carry. Often, these states have lifetimes in the picosecond range, requiring kiloteslastrength fields to perform $g$-factor measurements. Such fields can only result from hyperfine interactions. Two useful hyperfine fields are the transient field [1], resulting from the interaction between a ferromagnetic solid and a swift ion traversing it, and the hyperfine field produced by the electron cloud surrounding the nucleus of a free ion [2]. In the late 1960s, Goldring et al. characterised the hyperfine interactions of ions that had recoiled into vacuum or low-density gas at velocities of a few percent of the speed of light [3]. The hyperfine interaction, which depends on the $g$ factor, perturbs the distribution of $\gamma$ rays from the nuclei. Thus, the $g$ factor can be determined. After the transient-field effect was discovered, however, it became the primarily used method to measure the $g$ factors of short-lived states from the mid-1970s onward.

The hyperfine-field method was largely neglected until 2005, when Stone et al. used the recoil-in-vacuum (RIV) technique to measure $g\left(2_{1}^{+} ;{ }^{132} \mathrm{Te}\right)$ using a radioactive ion beam (RIB) [4]. The RIV technique allows the unreacted radioactive beam to travel out of view of the $\gamma$-ray detectors, avoiding the accumulation of background radiation (a major problem for transient-field measurements [4]). Additionally, modern detector arrays allow for the coverage of a large solid angle. The $g$ factor was

\footnotetext{
*e-mail: u5600477@anu.edu.au

**e-mail: andrew.stuchbery@anu.edu.au
}

determined by calibrating the hyperfine interaction with the known $g$ factors and lifetimes of even-even stable Te isotopes. The success of this approach led to several RIB RIV measurements on nearby nuclides [5--7]. It is also possible to determine $g$ factors based on calculated hyperfine-field strengths. By performing a time-dependent (TD) RIV measurement, the nuclear precession frequency resulting from a simple hyperfine interaction can be measured directly. This technique was applied in the measurement of $g\left(2_{1}^{+} ;{ }^{24} \mathrm{Mg}\right)$ [8], which produced a precise value due to bare and $\mathrm{H}$-like charge states being dominant, making for a straight-forward analysis of the single, well-known hyperfine field. However, as higher- $Z$ nuclei are considered, the H-like (single electron) interaction becomes too high in frequency to resolve its time dependence. To reduce the measured hyperfine-field strength into the regime where its frequency can again be resolved requires measurements on multi-electron ions, instead. The challenge to calculate the relevant hyperfine interactions then becomes more complex. Measurements on some multi-electron systems neighbouring each other have produced results that are difficult to interpret [2], but imply that a few low-excitation atomic configurations must be dominant. To tackle the challenge of identifying these important atomic configurations, we model the hyperfine interaction by performing atomic structure calculations and evaluating the effect of atomic transitions in an ensemble of charge states by the Monte-Carlo method [9, 10].

The Monte-Carlo method is performed by populating a large number of atomic states across the relevant chargestate distribution and then simulating their decays. The contribution of each individual decay path is then averaged. A similar approach has been applied by Chen et al. [11], but it was not applied to time-dependent measurements. It also did not allow the initial distribution of 
atomic states to be parametrized. In this work, a modified approach is presented for the analysis of TDRIV data. Data taken on the ${ }^{56} \mathrm{Fe} 21_{1}^{+}$state are used to demonstrate the analysis, and a tentative value for $g\left(2_{1}^{+} ;{ }^{56} \mathrm{Fe}\right)$ is deduced.

\section{Methods}

\subsection{The ${ }^{56} \mathrm{Fe}$ time-differential recoil-in-vacuum measurement}

A TDRIV experiment was conducted at the ALTO facility at the IPN, Orsay, in which a $130 \mathrm{MeV}{ }^{56} \mathrm{Fe}$ beam was incident upon a target having $230 \mu \mathrm{g} / \mathrm{cm}^{2}$ of carbon on a $0.5 \mu \mathrm{m}$-thick nickel foil. Beam particles were excited on the carbon layer and recoiled out of the nickel. The OUPS plunger device [12] was used to detect forwardscattered carbon ions, and recoiling beam particles were stopped in a thick nickel foil $\left(5.8 \mathrm{mg} / \mathrm{cm}^{2}\right)$. The OUPS is capable of adjusting the foil position along the beam axis in order to perform a time-dependent measurement. Coincident $\gamma$ rays were detected at forward angles by the ORGAM [13], and backward angles by the MINIBALL [14] arrays. The charge-state distributions of ${ }^{56} \mathrm{Fe}$ ions traversing nickel foils at various energies were measured at the Australian National University (ANU) Heavy Ion Accelerator Facility (HIAF) [15].

\subsection{Hyperfine interaction in ions recoiling in vacuum}

The RIV technique can be used to measure the nuclear $g$ factor via a perturbation of the $\gamma$-particle angular correlation arising from the nuclear-electronic dipole interaction [3]. When ions recoil in vacuum the electron spins $(J)$ are presumed to have no preferred orientation in space, whereas the nuclear spins $(I)$ have been oriented by the Coulomb excitation. The nuclear and electron spins then couple and, in the vector model picture, begin to precess about their total spin $(F)$ at a frequency proportional to the $g$ factor. This interaction is illustrated in Fig. 1. For a single, static atomic state, the periodic de-alignment and

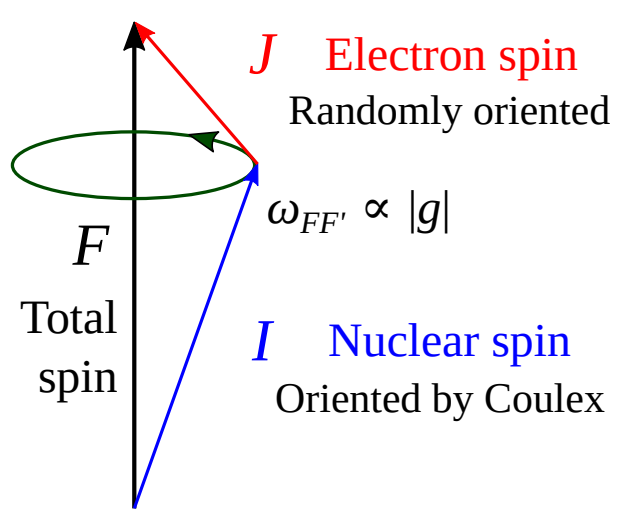

Figure 1. Spin coupling between the nuclear $(I)$ and electron $(J)$ spins, combining such that $F=I+J$. The dipole interaction results in a precession about $F$ with angular frequency $\omega_{F F^{\prime}}$ [see Eq. [2]]. re-alignment of the nuclear spin perturbs the angular correlation. This appears as an alternate attenuation and restoration in the anisotropy of the angular correlation as a cosine function. The periodic attenuation factor [3] is

$$
G_{k}(t)=\sum_{F, F^{\prime}} \frac{(2 F+1)\left(2 F^{\prime}+1\right)}{2 J+1}\left\{\begin{array}{ccc}
F & F^{\prime} & k \\
I & I & J
\end{array}\right\}^{2} e^{-i \omega_{F F^{\prime}} t}
$$

where $t$ is time, the quantum spin numbers have their established designation, the curly braces signify a Wigner $6-J$ symbol, and the precession frequency is

$$
\omega_{F F^{\prime}}=\frac{g B_{H F}}{2 J} \frac{\mu_{N}}{\hbar}\left[F(F+1)-F^{\prime}\left(F^{\prime}+1\right)\right] .
$$

where $B_{H F}$ is the hyperfine-field strength (which can be calculated from atomic theory), and $\mu_{N}$ is the nuclear magneton. Note that $w_{F F^{\prime}}$ varies for each $F, F^{\prime}$ coupling, resulting in a superposition of different frequencies when $J>1 / 2$.

When multiple atomic states contribute in sequence, their effect is multiplicative. Alignment lost through precession of a prior state is never regained, fixing a new maximum alignment. The average attenuation coefficient for an individual charge state $G_{k}^{A Q}$, resulting from atomic decays through a sequence of states, is thus

$$
G_{k}^{A Q}\left(t_{N}\right)=G_{k}^{1}\left(t_{1}\right) \prod_{i=2}^{N} G_{k}^{(i)}\left(t_{i}-t_{i-1}\right) .
$$

where $A$ and $Q$ represent the atomic-decay sequence and charge state, respectively, $G_{k}^{(i)}$ is the attenuation coefficient in state $i, t_{i}$ is the time at which the state decayed, and $N$ is the total number of states in the decay cascade up to time $t_{N}$.

Generally, there will be a distribution of charge states, each contributing a large number of atomic decay sequences with their own resulting $G_{k}^{A Q}$. Hence, the average contribution of $G_{k}^{A Q}$ for each charge state must be considered. The value obtained when averaging across a chargestate distribution $\bar{G}_{k}$ is determined by

$$
\bar{G}_{k}(t)=\sum_{Q}^{N_{Q}} c_{Q} \sum_{A}^{N_{A}} G_{k}^{A Q}(t) / N_{A} .
$$

where $N_{A}$ is the number of atomic-decay chains in an individual charge state, $N_{Q}$ is the number of charge states, and $c_{Q}$ is the fractional population of an individual charge state.

\subsection{Attenuation of $\gamma$-particle angular correlations}

The perturbed $\gamma$-ray angular distributions from oriented nuclei can be used to perform $g$-factor measurements [16]. When an excited $2^{+}$state is populated via Coulomb excitation the statistical tensor defining the orientation of the state can be calculated from semi-classical electromagnetic theory [17]. It is determined largely by the experimental particle-detection geometry, with little sensitivity 
to the $E 2$ matrix elements. The angular correlation [18] can be calculated by

$$
W(t, \phi, \theta)=\sum_{k, q} \sqrt{2 k+1} \rho_{k q} G_{k}(t) F_{k} Q_{k} D_{q 0}^{k *}(\phi, \theta, 0) .
$$

where $k=0,2,4$ and $-k \leq \mathrm{q} \leq \mathrm{k}, \rho_{k q}$ is the statistical tensor defining the spin alignment of the excited nuclear state, $G_{k}(t)$ is the attenuation coefficient, $F_{k}$ represents the $F$ coefficients for the $\gamma$-ray transition [17], $Q_{k}$ is the attenuation factor due to the finite $\gamma$-ray detector size, and $D_{q 0}^{k}$ is the Wigner $D$ rotation matrix [17]. The angles $\phi$ and $\theta$ are spherical polar coordinates, where the position of the beam spot on the target represents the origin, and the beam direction defines the $z$ axis. $\phi$ is the relative azimuthal angle between the $\gamma$-ray detector and the particle detector, and $\theta$ is the polar angle of the $\gamma$-ray detector.

The time-dependent attenuation coefficient, $G_{k}(t)$, can be measured using a plunger device, which quenches the hyperfine interaction at a particular distance (i.e after a set flight time) by implantation into a foil [19]. By measuring $\gamma$-particle coincidence events using a segmented, annular particle detector, $\theta$ - and $\phi$-dependent angular correlations can be measured as a function of time. $G_{k}(t)$ values can be obtained by fitting Eq. (5) to the data, with $G_{k}(k=2,4)$ as free parameters $\left(G_{0}=1\right)$. The $G_{k}$ values can also be calculated a priori, making the $g$ factor the only unknown parameter. Atomic states having spin $J=1 / 2$ are best suited to measure $g$ factors as they have a single cosine frequency over the summation of $F, F^{\prime}$. In our case Na-like ions, having $J=1 / 2$ ground states, are the most useful charge state to populate.

\subsection{Atomic structure calculations}

To model the hyperfine interaction, the atomic structure information of the charge states must be obtained. Progress in the field of atomic physics allows the calculation of atomic wavefunctions with great accuracy. One particular solution for calculating atomic properties, the General Relativistic Atomic Structure Package (GRASP), is freely available under the MIT license [20]. GRASP numerically solves the multiconfiguration Dirac-HartreeFock equations for atomic-state functions (ASFs) to obtain radial wavefunctions. AFSs are composed of a mixture of configuration-state functions (CSFs), defined by individual electron configurations. The vital point in using GRASP is understanding how the ASF is constructed. This is calculated by

$$
\Psi(\gamma P J)=\sum_{i=1}^{N} c_{i} \Phi\left(\gamma_{i} P J\right) .
$$

where $\Psi$ is the ASF representing a physical atomic state $\gamma$ having parity $P$ and angular momentum $J, N$ is the number of chosen CSFs, with each CSF $i$ having a mixing coefficient $c_{i}$, and a Slater determinant $\Phi$ defined by the electron configuration $\gamma_{i}$, parity $P$ and angular momentum $J$.

Equation (6) shows that any CSF having the same parity $(P)$ and angular momentum $(J)$ can contribute to a given ASF. To obtain the most accurate solution, one would include CSFs up to the continuum states. However, this is impractical as convergence becomes more difficult and computation time becomes longer as the number of CSFs increases. As such, one should choose CSFs that will have a significant $c_{i}$. Because we aim to simulate spectral cascades, our approach has been to calculate ASFs having leading terms that are valence configurations. The CSFs chosen for these calculations allowed for double excitations from the core electrons, having principal quantum number $n=2$ or $n=1$ in this case, to improve transition-rate calculations. A single-electron excitation from the deep core $(n=1)$ electrons was also allowed, to improve the hyperfine-field-strength calculation. CSFs were taken up to a value of $n$ where their number exceeded $\sim 10^{6}$, at which point computation time was already quite long ( $\sim 1$ week). The CSF space was increased by intervals of $n$, with each new value adding a new electron shell. The ASFs were resticted to energy levels below $1000 \mathrm{eV}$, as per Ref. [10]. Once solutions were obtained, the calculated energy levels were compared to those in the US National Institute of Standards and Technology database for atomic spectra [21], and found to agree better than $1 \%$ in all cases, and $0.1 \%$ in most. This comparison is helpful in determining whether the radial wavefunctions have converged on an accurate solution. Another way convergence was assessed was to compare the results of the two oscillator strength calculation approaches (alternate forms for the dipole operator, or gauges) GRASP takes, namely the length and velocity forms [22]. Disagreement indicates the solution is not self-consistent. Most transitions were found to agree better than $1 \%$, with poor agreement only observed for slow transitions between high-energy states, which were deemed unimportant in the present context due to the unlikeliness of their population or observation.

\subsection{Monte-Carlo simulation}

Finding an exact mathematical solution to the complex problem of calculating $\bar{G}_{k}(t)$ is not practical. Instead, the Monte-Carlo method is used to obtain a solution by conducting a "virtual experiment" using the atomic structure calculations. For a given species an initial atomic state is selected at random following the chosen energy distribution (e.g. Boltzmann or uniform). A nuclear survival time for the event is then generated by

$$
t_{N}\left(\tau_{N}\right)=-\log _{e}(P) \times \tau_{N} .
$$

where $\tau_{N}$ is the nuclear-state lifetime, and $P$ is a random number evenly distributed between $(0,1]$. Then, the following procedure is iterated (after initialising the cumulative atomic survival time $t_{c}$ to zero) :

1. If the atomic lifetime $\left(\tau_{a}\right)$ is finite, generate an atomic survival time $\left(t_{a}\right)$ for the atomic state as per Eq. (7), but with $\tau_{a}$ in place of $\tau_{N}$. Otherwise, set $t_{a}=t_{N}-t_{c}$ and end the event.

2. If the cumulative and atomic survival time exceed the nuclear survival time $\left(t_{c}+t_{a}>t_{N}\right)$ then set 
$t_{a}=t_{N}-t_{c}$ and end the event. Otherwise, add $t_{a}$ to $t_{c}$.

3. Select a new atomic state based on the transition probabilities.

Atomic levels and $t_{a}$ values are recorded for each iteration. This procedure produces an event consisting of a number of atomic-level references and survival times, with a cumulative atomic survival time equal to the nuclear survival time. This information is then used to evaluate the atomicstate population through time, and once that is performed for each charge state, to calculate $\bar{G}_{k}(t)$.

\section{Results and Discussion}

\subsection{Charge-state distribution}

Figure 2 shows the expected charge-state distribution of ${ }^{56} \mathrm{Fe}$ ions in the ALTO measurement, centred on Na-like ions. The distribution is quite broad, with the neighbouring $\mathrm{Ne}$ - and $\mathrm{Mg}$-like species each comprising 70\% of the Na-like population. In terms of atomic excitation, the Nelike ground state will be dominantly occupied due to the closed electron shell. Having $J=0$, there will be no net hyperfine interaction. The effect of this state on $\bar{G}_{k}(t)$ will be to reduce the amplitude of the Na-like $J=1 / 2$ cosine frequency. The Mg-like species will have many populated excited atomic states, close in energy, due to having two valence electrons. The hyperfine interaction in such species appears as a quasi-exponential attenuation through time, again effectively reducing the Na-like cosine frequency amplitude. The effect is the same for the Al-like charge state. The single-hole F-like ions have low-energy, longlived atomic states with hyperfine-field strengths similar to the Na-like atomic states. If they are strongly populated, the resulting interference patterns may be difficult to deconvolute.

\subsection{Atomic-state populations}

Monte-Carlo simulations, using the calculated atomic energy levels and transition rates, were used to explore how the population of atomic states could vary through time.

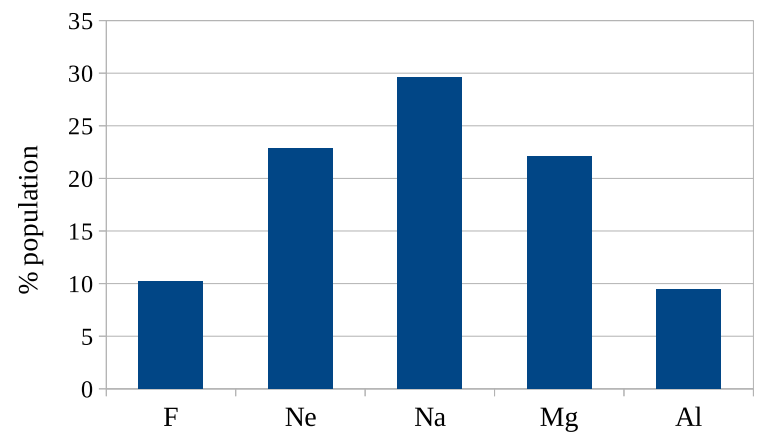

Figure 2. Estimated charge-state distribution of ${ }^{56} \mathrm{Fe}$ ions in the TDRIV measurement at ALTO.
These populations obviously depend on the initial distribution of atomic states, which is not well known. A comparison of the atomic population distributions for Na-like ${ }^{56} \mathrm{Fe}$ ions with time, one having an initial population with a Boltzmann energy distribution of mean energy $T=100 \mathrm{eV}$, and the other a uniform distribution up to the highestenergy ASF, is shown in Fig. 3 Both initial distributions populate similar atomic states at longer times $(>25 \mathrm{ps})$, but have different relative intensities on the order of the nuclear lifetime (10 ps). The initial Boltzmann distribution results in low-energy states being preferentially populated from early time points, with the ground state having a dominant contribution from $t=0$. Feeding cascades from higher-energy states are also much less influential.

Of the five charge states present, only two have atomic states with observable frequencies: Na-like and F-like. Figure 3 shows that irrespective of whether a Boltzmann or uniform initial distribution is chosen, for Nalike ions three low-lying atomic states, $3 s_{1 / 2}(0), 3 p_{1 / 2}(1)$ and $3 d_{5 / 2}(4)$, where the numbers in parentheses are the state labels in Fig. 3, are rapidly $(<5 \mathrm{ps})$ populated. Of these three, only the $3 s_{1 / 2}$ and $3 p_{1 / 2}$ states have observable frequencies. In F-like ions, proximity to the neon shell-gap results in the ground and first-excited state $\left(2 p_{3 / 2}\right.$ and $2 p_{1 / 2}$ holes) being dominantly populated within a few picoseconds, and both have observable frequencies. For

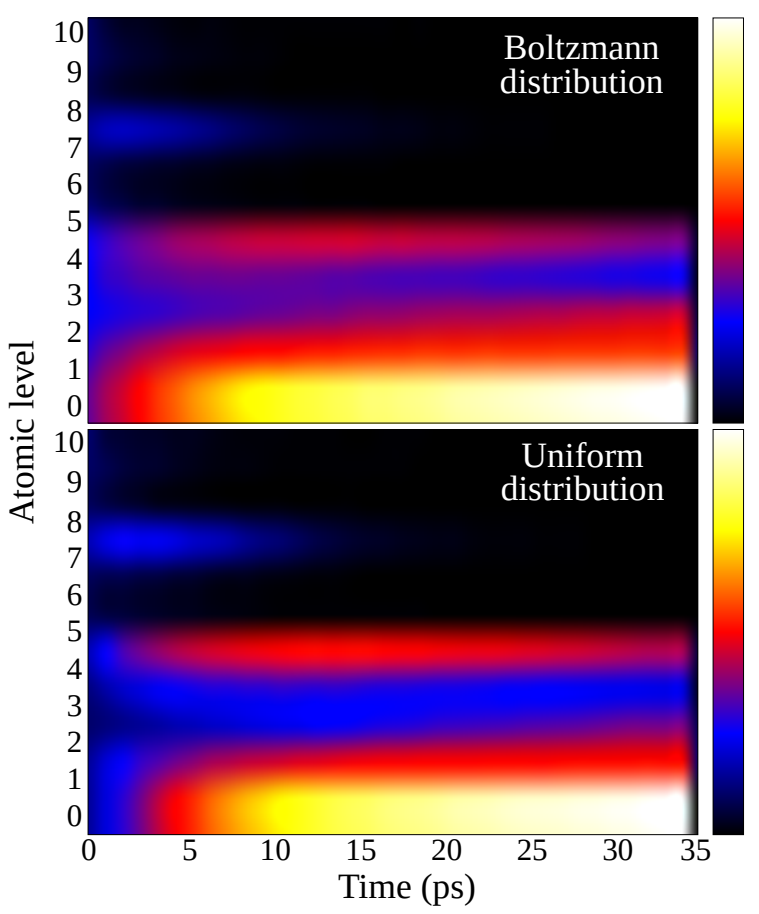

Figure 3. Monte-Carlo simulation of Na-like atomic-state distributions for Fe ions, showing an initial Boltzmann $(T=100 \mathrm{eV})$ vs uniform distribution. The $y$ axis shows the energy-ordered level index. The temperature scale is set relative to the most intense population on the plot. Higher-excited states, which feed the lower states, are included in these calculations but not shown. Note that due to binning and fast transitions, the $\mathrm{t}=0$ distribution does not show the true initial distribution shape. 


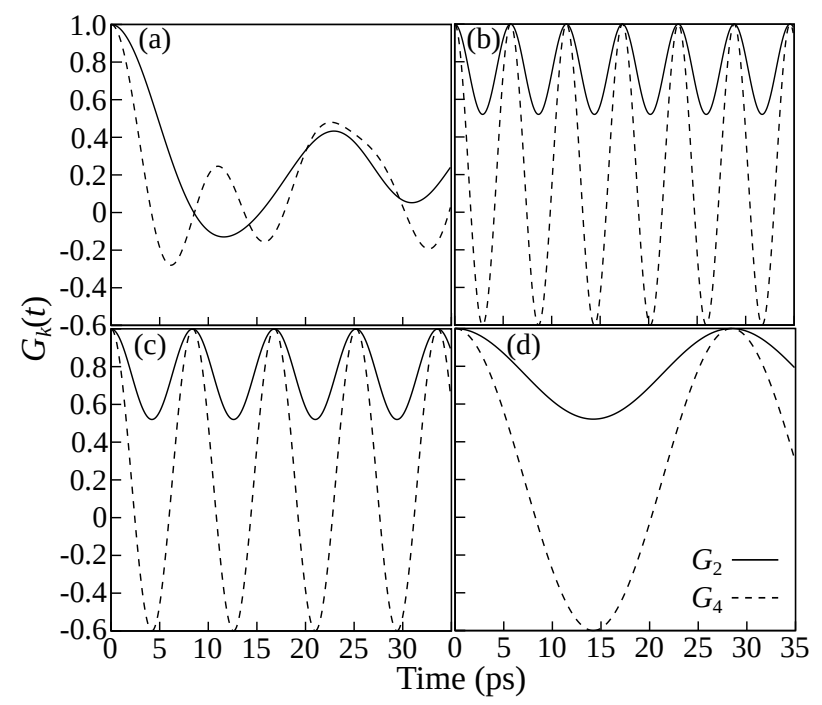

Figure 4. Individual $G_{k}(t)$ plots for Fe ion (a) F-like $2 p_{3 / 2}$, (b) F-like $2 p_{1 / 2}$, (c) Na-like $3 s_{1 / 2}$, and (d) Na-like $3 p_{1 / 2}$. These are all evaluated with $g\left(2_{1}^{+} ; \mathrm{Fe}^{56}\right)=0.51$, as previously reported [23].

these four atomic states, the pure $G_{k}$ vs $t$ plots of their individual frequencies are shown in Fig. 4

\subsection{Fitting the $g$ factor}

Measured $G_{k}(t)$ values were obtained by fitting Eq. (5) to the data as described in Section 2.3. For clarity of analysis, a subset of the total data was chosen. This subset was taken by the ORGAM array (having high statistics) and rejected $\gamma$-rays emitted by nuclei which had decayed in flight. By varying the $g$ factor used in the calculation of $\bar{G}_{k}(t)$ from the Monte-Carlo simulation events, an optimal fit to the data points can be found. Another important fit parameter is the distribution of atomic states, as this will affect the observed frequencies and the average magnitude of the $\bar{G}_{k}$ values. Presently, this parameter is poorly understood. Because of this, it is illuminating to instead consider, individually, the major contributing atomic states, and whether they have frequencies that will appreciably affect the measurement. The atomic-state-population heatmaps (Fig. 3), instead of reproducing the measured data, were used to help identify significantly contributing states. A frequency superposition of these states then allowed grand features of the measured $G_{k}(t)$ values to be probed by varying their population. If these features of the $G_{k}$ vs $t$ plot can be unambiguously reproduced, then the $g$ factor can be robustly determined.

The measured $G_{k}(t)$ values are shown in Fig. 5 along with a fit using only the frequencies shown in Fig. 4 Focusing on the experimental data points, there are two notable features: the peak-like increase in $G_{k}$ at $16 \mathrm{ps}$, and the broader increase at $25 \mathrm{ps}$. By reference to Fig. 4 . the interference pattern between the F-like $2 p_{1 / 2}$ and the Na-like $3 s_{1 / 2}$ frequencies produces a constructive peak at $16 \mathrm{ps}$, but interferes more destructively elsewhere. This near-cancellation can explain the smoother variation from 6 to $10 \mathrm{ps}$, and 23 to $27 \mathrm{ps}$. Both the F-like $2 p_{3 / 2}$ and the
Na-like $3 p_{1 / 2}$ frequencies can contribute to the increase in $G_{k}$ at $25 \mathrm{ps}$, but the Na-like $3 p_{1 / 2}$ frequency appears to be dominant. Additionally, the different effective frequencies for $G_{2}$ and $G_{4}$ from the F-like $2 p_{3 / 2}$ state, resulting from its $J=3 / 2$ atomic spin, can explain the proximity of $G_{2}$ and $G_{4}$ values near $10 \mathrm{ps}$ and $20 \mathrm{ps}$. The frequency superposition in Fig. 5 was made to be in line with the measured charge state distribution and predicted atomic-state populations, and to reproduce the peaks at $16 \mathrm{ps}$ and at 25 ps. Fitting these features gives $g \approx 0.55$, different from the literature value indicated in Fig. 4, but consistent with it, within experimental uncertainty. Changing $g$ by more than \pm 0.02 causes significant misalignment of the fit from these prominent features.

When attempting to fit the entire timespan, the data seem to show a changing relative population of states through time. Initially, a strong relative contribution from the F-like states matches the initial rapid decrease, with the $2 p_{3 / 2}$ state's frequency responsible for the proximity of $G_{2}$ and $G_{4}$ around $10 \mathrm{ps.} \mathrm{After} \mathrm{this} \mathrm{point,} \mathrm{increasing}$ population of the Na-like states relative to the F-like states matches the sharp peak at $16 \mathrm{ps}$, and their continued population increase is required to bring about the large peak at $25 \mathrm{ps}$. A plausible explanation is that, initially, higherenergy excited atomic states are strongly populated in the Na-like ions, and then decay to the low-energy states. However, Monte-Carlo simulations have so far been unable to accurately reproduce this behaviour. This hypothesis is currently under investigation. The complicated superposition of changing populations makes a global fit difficult. Preliminary results from a global fit indicate that the $g$ factor could possibly be determined with an uncertainty below \pm 0.01 . It should be noted, however, that systematic sources of uncertainty are yet to be quantified, with the

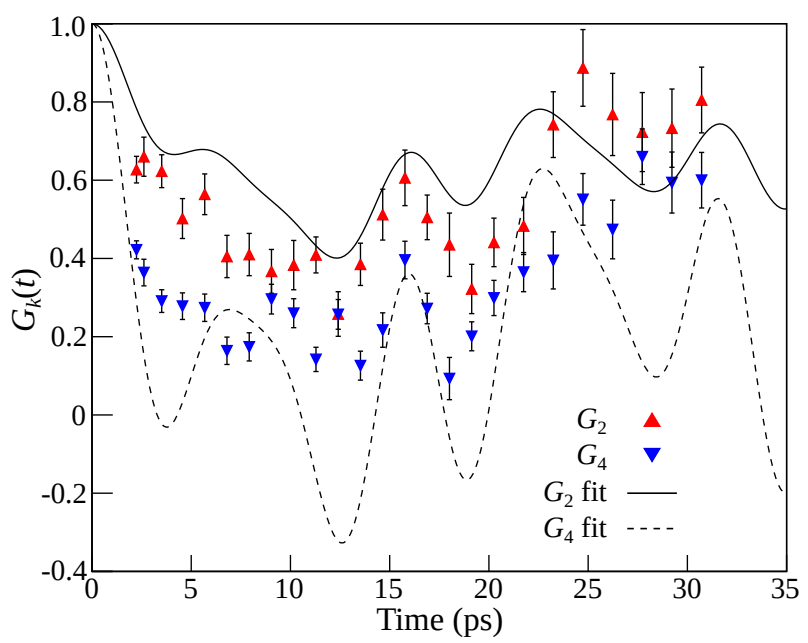

Figure 5. $G_{k}$ vs $t$ plot showing data points obtained by freefitting $G_{2}$ and $G_{4}$ to measured angular correlations (as described in Section 2.3, and a superposition of the frequencies shown in Fig. 4 having relative populations of $25 \%$ F-like $2 p_{3 / 2}, 15 \%$ Flike $2 p_{1 / 2}, 35 \%$ Na-like $3 s_{1 / 2}$ and $25 \%$ Na-like $3 p_{1 / 2}$. For the frequencies shown here, $g=0.55$, obtained from producing an optimal fit the values forming the peak at $16 \mathrm{ps}$. 
most significant expected to be the uncertainty in the absolute time offset (plunger zero-distance). However, even at this stage of analysis, fitting of major trends can evidently provide a robust measure of the $g$ factor.

It should be clarified that $G_{2}$ and $G_{4}$ are highly correlated, so free-fitting by $\chi^{2}$ minimisation may not give correct relative magnitudes. It will, however, reveal frequency trends that are useful in guiding the data analysis. As such, discrepancies in the magnitude of the fitted vs. measured values should not be considered too harshly at this preliminary stage. An approach by which $G_{k}$ values are calculated a priori and fitted directly to the angular correlation, while also allowing atomic-state populations to vary in time realistically, is in progress.

\section{Conclusion}

The TDRIV measurement technique has been demonstrated as a promising way to measure $g\left(2_{1}^{+}\right)$values in short-lived nuclear states of radionuclides. Detailed Monte-Carlo simulations based on atomic-structure calculations reveal that multiple atomic states contribute to the hyperfine interaction for nuclei around $\mathrm{Z}=30$, creating a superposition of frequencies. By modelling and fitting the population of states through time, it appears that the $g$ factor can be reliably determined. The relevant hyperfine interaction frequencies from a TDRIV data set on ${ }^{56} \mathrm{Fe}$ were identified and suggest $g \approx 0.55$, with an accuracy of $\sim 4 \%$. An improved data analysis technique is being developed, from which preliminary results indicate that the $g$ factor could be determined with close to $1 \%$ precision, the final precision likely dependent on systematic uncertainties associated with the plunger technique as much as the modelling of the hyperfine fields.

\section{Acknowledgements}

The authors are grateful to the academic and technical staff of the IPN ALTO facility and the ANU HIAF for their assistance and maintenance of the facilities. In particular, the authors would like to thank J. Ljungvall, I. Matea, T. Konstatinopoulos, K. Gladnishki, A. Gottardo and D. Yordanov for their assistance with the data collection. This research was supported in part by the Australian Research Council under grant number DP170101673. A. G. acknowledges the support of the P2IO Excellence Laboratory. B. P. M. acknowledges the support of the Australian Government Research Training Program. Support for the ANU Heavy Ion Accelerator Facility operations through the Australian National Collaborative Research Infrastructure Strategy (NCRIS) program is acknowledged.

\section{References}

[1] N. Benczer-Koller and G. J. Kumbartzki, Journal of Physics G: Nuclear and Particle Physics 34, R321 (2007).

[2] A. E. Stuchbery, Hyperfine Interactions 220, 29 (2013).

[3] G. Goldring, in Heavy Ion Collisions, edited by R. Bock (North-Holland Pub. Co., 1982), Vol. 3, p. 483.

[4] N. J. Stone, A. E. Stuchbery, M. Danchev, et al., Phys. Rev. Lett. 94, 192501 (2005).

[5] J. M. Allmond, A. E. Stuchbery, D. C. Radford, et al., Phys. Rev. C 87, 054325 (2013).

[6] A. E. Stuchbery, J. M. Allmond, A. Galindo-Uribarri, et al., Phys. Rev. C 88, 051304(R) (2013).

[7] A. E. Stuchbery, J. M. Allmond, M. Danchev, et al., Phys. Rev. C 96, 014321 (2017).

[8] A. Kusoglu, A. E. Stuchbery, G. Georgiev, et al., Phys. Rev. Lett. 114, 062501 (2015).

[9] A. E. Stuchbery and N. J. Stone, Phys. Rev. C 76, 034307 (2007).

[10] N. J. Stone, J. R. Stone and P. Jönsson, Hyperfine Interactions 197, 29 (2010).

[11] X. Chen, D. G. Sarantites, W. Reviol and J. Snyder, Phys. Rev. C 87, 044305 (2013).

[12] J. Ljungvall, G. Georgiev, S. Cabaret, et al., Nucl. Inst. Meth. A 679, 61 (2012).

[13] C. Le Galliard, Tech. rep., IPN Orsay, IPN Orsay, University of South Paris, Orsay, France (2019), http: //ipnwww . in2p3. fr/Orgam?lang=en.

[14] N. Warr, J. Eberth, G. Pascovici, et al., MINIBALL: A Gamma-ray spectrometer for exotic beams (2003).

[15] A.E. Stuchbery, A.B. Harding, D.C. Weisser, N.R. Lobanov, Nucl. Instrum. Methods Phys. Res. A 951, 162985 (2020).

[16] A. E. Stuchbery and M. P. Robinson, Nucl. Instrum. Meth. Phys. Res. A 485, 753 (2002).

[17] K. Alder, A. Winther, Electromagnetic excitation: theory of Coulomb excitation with heavy ions (Amsterdam: North Holland, 1975).

[18] A. E. Stuchbery, Nuclear Physics A 723, 69 (2003).

[19] A. Kusoglu, A.E. Stuchbery, G. Georgiev, et al., J. Phys. C 590, 012041 (2015).

[20] C. Froese-Fischer, G. Gaigalas, P. Jönsson, J. Bieroń, Computer Physics Communications 237, 184 (2019).

[21] Y. Ralchenko, NIST Atomic Spectral Database pp. https://www.nist.gov/pml/atomic-spectra-database (2019).

[22] C. Froese-Fischer, T. Brage and P. Jönsson, Computational Atomic Structure: An MCHF Approach (Inst. Phys. Pub. Bristol and Philadelphia, 1997).

[23] M. C. East, A. E. Stuchbery, S. K. Chamoli, et al., Phys. Rev. C 79, 024303 (2009). 